\title{
Isogeometric shape optimization in fluid mechanics
}

\author{
Nørtoft, Peter; Gravesen, Jens
}

Published in:

Structural and Multidisciplinary Optimization

Link to article, DOI:

10.1007/s00158-013-0931-8

Publication date:

2013

Document Version

Peer reviewed version

Link back to DTU Orbit

Citation (APA):

Nørtoft, P., \& Gravesen, J. (2013). Isogeometric shape optimization in fluid mechanics. Structural and Multidisciplinary Optimization, 48(5), 909-925. https://doi.org/10.1007/s00158-013-0931-8

\section{General rights}

Copyright and moral rights for the publications made accessible in the public portal are retained by the authors and/or other copyright owners and it is a condition of accessing publications that users recognise and abide by the legal requirements associated with these rights.

- Users may download and print one copy of any publication from the public portal for the purpose of private study or research.

- You may not further distribute the material or use it for any profit-making activity or commercial gain

- You may freely distribute the URL identifying the publication in the public portal

If you believe that this document breaches copyright please contact us providing details, and we will remove access to the work immediately and investigate your claim. 


\title{
Isogeometric Shape Optimization in Fluid Mechanics
}

\author{
Peter Nørtoft • Jens Gravesen
}

\begin{abstract}
The subject of this work is numerical shape optimization in fluid mechanics, based on isogeometric analysis. The generic goal is to design the shape of a 2dimensional flow domain to minimize some prescribed objective while satisfying given geometric constraints. As part of the design problem, the steady-state, incompressible Navier-Stokes equations, governing a laminar flow in the domain, must be solved. Based on isogeometric analysis, we use B-splines as the basis for both the design optimization and the flow analysis, thereby unifying the models for geometry and analysis, and, at the same time, facilitating a compact representation of complex geometries and smooth approximations of the flow fields. To drive the shape optimization, we use a gradient-based approach, and to avoid inappropriate parametrizations during optimization, we regularize the optimization problem by adding to the objective function a measure of the quality of the boundary parametrization. A detailed description of the methodology is given, and three different numerical examples are considered, through which we investigate the effects of the regularization, of the number of geometric design variables, and of variations in the analysis resolution, initial design and Reynolds number, and thereby demonstrate the robustness of the methodology.
\end{abstract}

Keywords shape optimization - isogeometric analysis · fluid mechanics - regularization - Navier-Stokes equation $\cdot$ Taylor-Couette flow $\cdot$ drag

P. Nørtoft · J. Gravesen

DTU Compute, Technical University of Denmark, Matematiktorvet 303B, DK-2800 Kgs. Lyngby

Tel.: $+45-4525-3031$

Fax: $+45-4588-1399$

E-mail:penn@dtu.dk (P.Nørtoft)

E-mail:jgra@dtu.dk (J. Gravesen)

\section{Introduction}

Numerical shape optimization in fluid mechanics is the art of using computers to find "best" shapes in engineering problems involving flows, based on some notion of "goodness" (Mohammadi and Pironneau 2010), and applications ranges from, e.g., microfluidic proteinfolding devices (Ivorra et al 2006) to airplane wings (Painchaud-Oullet et al 2006). Isogeometric analysis is a recently proposed computational methodology for solving engineering problems (Hughes et al 2005; Cottrell et al 2009). It unites the analysis powers from finite element analysis (FEA) in terms of solving partial differential equations with the powers from computer aided design (CAD) in terms of geometric modeling. The focus of this study is the use of isogeometric analysis for numerical shape optimization in fluid mechanics.

From a fluid mechanics point of view, isogeometric analysis is appealing in particular due its ability to represent complex geometries in few variables (Bazilevs and Hughes 2008), and because of the inherent high regularity of the flow fields (Akkerman et al 2010). From a shape optimization point of view, isogeometric analysis serves as a natural framework, due to its ability to represent complex shapes in few design variables, and its tight connection between analysis and geometry models. This means that an accurate representation of the geometry can be maintained throughout the optimization, and there is no need of communication between FEA and CAD models (Wall et al 2008).

In recent years, isogeometric analysis has successfully been applied to various shape optimization problems in mechanical engineering. Numerous studies within structural mechanics have been made, using either NURBS control points (Wall et al 2008; Cho and Ha 2009), NURBS control points and weights (Nagy et al 2010a,b; Qian 2010; Nagy et al 2011), or T-splines control points 
(Ha et al 2010; Seo et al 2010b) as design variables. NURBS-based isogeometric shape optimization using a boundary integral method has also been studied ( $\mathrm{Li}$ and Qian 2011). Applications of isogeometric shape optimization also include studies of vibrating membranes (Nguyen et al 2011), and of photonic crystals (Qian and Sigmund 2011), and worth mentioning are also recent applications of isogeometric topology optimization within structural mechanics (Seo et al 2010b,a; Hassani et al 2012).

An inherent challenge in numerical shape optimization is to maintain a high quality of the computational mesh as the shape of the domain changes during optimization (Mohammadi and Pironneau 2004; Bletzinger et al 2010). This also applies in an isogeometric approach. Here, the shape is given by control points, and in this setting, care has to be taken to avoid clustering and folding over of these control points during optimization, which in turn may lead to singular parametrizations (Wall et al 2008; Nagy et al 2011).

The aim of this work is twofold. Firstly, we present numerical investigations of how to apply isogeometric analysis as a framework for shape optimization in fluid mechanics, illustrating how the method may be used both as analysis tool to solve the Navier-Stokes equations, and as design tool to guide an optimization procedure through analytically computed gradients of objective and constraint functions, using B-spline control points as design variables. Secondly, to ensure appropriate parametrizations during the optimization, we construct a measure for regularization of the shape optimization problem, and based on a benchmark optimization problem, in which we design a pipe bend to minimize the pressure drop of the flow through it, we investigate how this regularization measure influences the optimization process and the optimal design. To investigate the robustness of the isogeometric shape optimization methodology, we apply it to two additional optimization problems in fluid mechanics. Firstly, to investigate the effect of the number of design control points, we design a body at rest in a circular fluid container with rotating boundary to obtain a uniform pressure distribution along its boundary, a design problem which happens to have a known solution. Secondly, to investigate the effects of variations in analysis mesh, initial design, and Reynolds number, we design a body traveling at constant speed through a fluid to minimize the drag, a classical shape optimization problem in fluid mechanics.

The outline of the paper is as follows: Section 2 introduces the generic shape optimization in fluid mechanics studied in the paper, and in Section 3 we present its isogeometric implementation. The regularization tech- nique is investigated in Section 4, after which two applications of the methodology are presented in Section 5. Finally, in Section 6, conclusions are drawn and a brief outlook is given.

\section{Shape Optimization Problem}

We consider a viscous, incompressible, isothermal, steady flow at low to moderate Reynolds numbers in a 2-dimensional domain $\Omega$ as depicted in Figure 1. The fluid is assumed

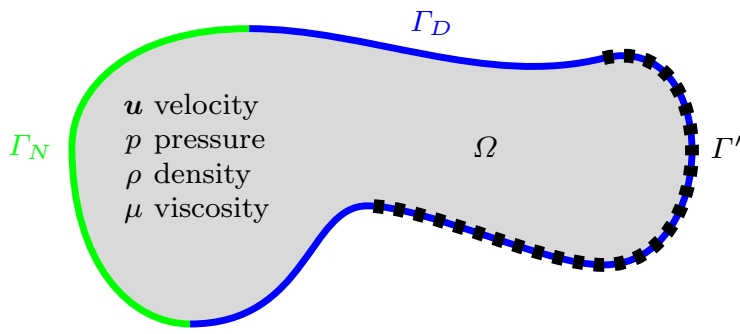

Fig. 1 Setup of the generic shape optimization problem in fluid mechanics

to be Newtonian with constant density $\rho$ and viscosity $\mu$, and the state of the fluid is characterized by its velocity $\boldsymbol{u}=\boldsymbol{u}(\boldsymbol{x})=(u(\boldsymbol{x}) v(\boldsymbol{x}))^{T}$ and its pressure

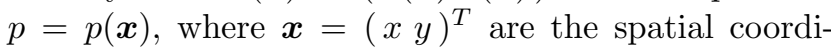
nates. We assume that no external body forces act on the system. For the boundary $\Gamma$, we assume that the domain is open along the Neumann part $\Gamma_{N}$, and that the flow field $\boldsymbol{u}$ is given along the Dirichlet part $\Gamma_{D}$, independently of the shape. The flow is then governed by:

$$
\begin{aligned}
\rho(\boldsymbol{u} \cdot \nabla) \boldsymbol{u}-\nabla p+\mu \nabla^{2} \boldsymbol{u} & =\mathbf{0} \\
\nabla \cdot \boldsymbol{u} & =0 \\
\left.\boldsymbol{u}\right|_{\Gamma_{D}} & =\boldsymbol{u}^{*} \\
\left.\left(\mu \nabla u_{i}-p \boldsymbol{e}_{i}\right) \cdot \boldsymbol{n}\right|_{\Gamma_{N}} & =0 .
\end{aligned}
$$

Here, Equations (1a) and (1b) are the Navier-Stokes equation and the incompressibility condition, respectively, governing the flow in the domain interior $\Omega$, while Equations (1c) and (1d) are the Dirichlet and the Neumann boundary conditions, respectively, where $\boldsymbol{u}^{*}$ is the given velocity field. Also, $\boldsymbol{e}_{i}$ is the orthonormal basis in $\mathbb{R}^{2}, i=1,2$ is the component index, and $\boldsymbol{n}$ is the outward unit normal.

The aim of the optimization is to design the shape of a specified part $\Gamma^{\prime}$ of the boundary of the domain to minimize a prescribed objective function, with a constraint on the area of the domain. The specific form of the objective function will be explained further below. 
The goal is thus to solve the following generic shape optimization problem:

$\underset{\Gamma^{\prime}}{\operatorname{minimize}} \quad C\left(\boldsymbol{u}\left(\boldsymbol{x}, \Gamma^{\prime}\right), p\left(\boldsymbol{x}, \Gamma^{\prime}\right), \Gamma^{\prime}\right)$

such that $A_{\min } \leq A\left(\Gamma^{\prime}\right) \leq A_{\max }$

$$
\mathcal{L}_{\text {min }} \leq \mathcal{L}\left(\Gamma^{\prime}\right) \leq \mathcal{L}_{\text {max }}
$$

where $\Gamma^{\prime}$ is the design boundary, Equations (2a) and (2b) are the objective and the area constraint functions, respectively, Equation (2c) establishes additional linear constraints as dictated by the geometry and the physics of the specific problem, and the velocity $\boldsymbol{u}\left(\boldsymbol{x}, \Gamma^{\prime}\right)$ and the pressure $p\left(\boldsymbol{x}, \Gamma^{\prime}\right)$ solve the governing equations (1).

We will consider three different quantities as the cost function $C$ in Equation (2a): the difference in mean pressure between two boundary segments $\gamma_{+}$and $\gamma_{-}$, the pressure variation along a boundary segment $\gamma$, and the aerodynamic drag on a boundary segment $\gamma$. These are given by:

$$
\begin{aligned}
C_{\Delta p} & =\frac{\int_{\boldsymbol{\gamma}_{+}} p \mathrm{~d} s}{L_{\gamma_{+}}}-\frac{\int_{\boldsymbol{\gamma}_{-}} p \mathrm{~d} s}{L_{\gamma_{-}}}, \\
C_{\nabla p} & =\int_{\boldsymbol{\gamma}}(\nabla p \cdot \boldsymbol{t})^{2} \mathrm{~d} s, \\
C_{d} & =\int_{\boldsymbol{\gamma}}\left(-p \boldsymbol{I}+\mu\left(\nabla \boldsymbol{u}+(\nabla \boldsymbol{u})^{T}\right)\right) \boldsymbol{n} \mathrm{d} s \cdot \boldsymbol{e}_{u},
\end{aligned}
$$

respectively, where $L_{\gamma}=\int_{\gamma} \mathrm{d} s$ denotes the length of the segment $\gamma, \boldsymbol{t}$ is the unit tangent vector, $\boldsymbol{e}_{u}$ the constant unit vector along a specified direction, and $\boldsymbol{I}$ the identity matrix. The context in which these three different cost functions may appear will be exemplified in Sections 4, 5.1, and 5.2, respectively.

\section{Isogeometric Method}

In this section, we explain how B-spline based isogeometric analysis may be applied to the shape optimization problem (2)-(3). The reader is referred to, e.g., (Piegl and Tiller 1995) for a thorough treatment of Bsplines and NURBS (Non-Uniform Rational B-splines), (Donea and Huerta 2003) for an introduction to finite element analysis of Navier-Stokes flows, (Cottrell et al 2009) for a thorough treatment of isogeometric analysis, (Nielsen et al 2011) for an introduction to its application to Navier-Stokes flow, and (Wall et al 2008) for an introduction to its application to shape optimization.

\subsection{B-splines and NURBS}

The building blocks of the method are B-splines and NURBS. To set the scene, and for later reference, we briefly revise the basic concepts of these functions.
Univariate B-splines $\mathcal{N}_{i}^{q}:[0,1] \rightarrow \mathbb{R}$ are piecewise polynomials defined recursively from a polynomial degree $q \in \mathbb{N}$ and a knot vector $\Xi=\left\{\xi_{1}, \ldots, \xi_{m}\right\}$ with $\xi_{i} \in \mathbb{R}$ for $i=1, \ldots, m$ :

$\mathcal{N}_{i}^{0}(\xi)=\left\{\begin{array}{l}1 \text { if } \xi_{i} \leq \xi<\xi_{i+1} \\ 0 \text { otherwise }\end{array}\right.$

for $q=0$, and

$\mathcal{N}_{i}^{q}(\xi)=\frac{\xi-\xi_{i}}{\xi_{i+q}-\xi_{i}} \mathcal{N}_{i}^{q-1}(\xi)+\frac{\xi_{i+q+1}-\xi}{\xi_{i+q+1}-\xi_{i+1}} \mathcal{N}_{i+1}^{q-1}(\xi)$

for $q=1,2, \ldots$ with $i=1, \ldots, m-q-1$. We use the unit parametric domain $\xi \in[0,1]$, and we assume open knot vectors, i.e., the boundary knots have multiplicity $q+1$ with $\xi_{1}=\xi_{2}=\ldots=\xi_{q+1}=0$ and $\xi_{m}=\xi_{m-1}=$ $\ldots=\xi_{m-q}=1$.

Bivariate tensor product B-splines $\mathcal{P}_{i, j}^{q, r}:[0,1]^{2} \rightarrow \mathbb{R}$ are defined from the univariate B-splines above:

$\mathcal{P}_{i, j}^{q, r}(\xi, \eta)=\mathcal{N}_{i}^{q}(\xi) \mathcal{M}_{j}^{r}(\eta)$

where $\mathcal{N}_{i}^{q}$ is the $i^{\text {th }}$ univariate B-spline with degree $q$ and knot vector $\Xi_{1}=\left\{\xi_{1}, \ldots, \xi_{m}\right\}$ in the parametric dimension $\xi$, and $\mathcal{M}_{j}^{r}$ is the $j^{\text {th }}$ univariate B-spline with degree $r$ and knot vector $\Xi_{2}=\left\{\eta_{1}, \ldots, \eta_{n}\right\}$ in the parametric dimension $\eta$.

Bivariate NURBS $\mathcal{R}_{i, j}^{q, r}:[0,1]^{2} \rightarrow \mathbb{R}$ are defined from the bivariate B-splines above, and the weights $W=\left\{w_{1,1}, \ldots, w_{N, M}\right\}$ with $w_{i, j} \in \mathbb{R}$ for $i=1, \ldots, m-$ $q-1$ and $j=1, \ldots, n-r-1$ :

$\mathcal{R}_{i, j}^{q, r}(\xi, \eta)=\frac{w_{i, j} \mathcal{P}_{i, j}^{q, r}(\xi, \eta)}{\sum_{k=1}^{m-q-1} \sum_{l=1}^{n-r-1} w_{k, l} \mathcal{P}_{k, l}^{q, r}(\xi, \eta)}$.

The construction of bivariate B-splines from a set of polynomial degrees and knots vectors is illustrated in Figure 2. This type of function will serve as basis for the subsequent analysis and optimization.

\subsection{Geometry Parametrization}

Using bivariate tensor product NURBS $\mathcal{R}_{i}$ as defined above, we construct a parametrization $\boldsymbol{X}:[0,1]^{2} \rightarrow \mathbb{R}^{2}$ of the physical domain $\Omega$, cf. Figure 3 :

$\boldsymbol{X}=\left(\begin{array}{ll}x & y\end{array}\right)^{T}=\sum_{i}^{N_{\mathrm{var}}^{g}} \underline{\boldsymbol{X}}_{i} \mathcal{R}_{i}^{g}$

where $\underline{\boldsymbol{X}}_{i}=\left(\underline{x}_{i} \underline{y}_{i}\right)^{T} \in \mathbb{R}^{2}$ are control points, and $N_{\text {var }}^{g}$ is the number of terms in the expansion. The superscript $g$ indicates that the functions refer to given knot 


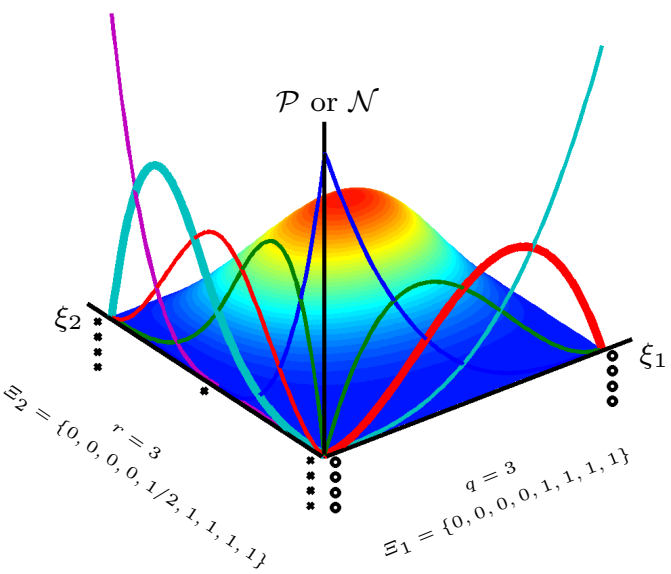

Fig. 2 Construction of a bivariate tensor product B-spline (surface) from two univariate B-splines (lines in bold) of given polynomial degrees and knot vectors (crosses and circles)

vectors, polynomial degrees and control weights specific for the geometry, and the subscript $i$ denotes a single vectorized index corresponding to the two indices used in Equation (6). The boundary of the flow domain is the image of the parameter boundaries: $\Gamma=\boldsymbol{X}\left(\partial[0,1]^{2}\right)$.

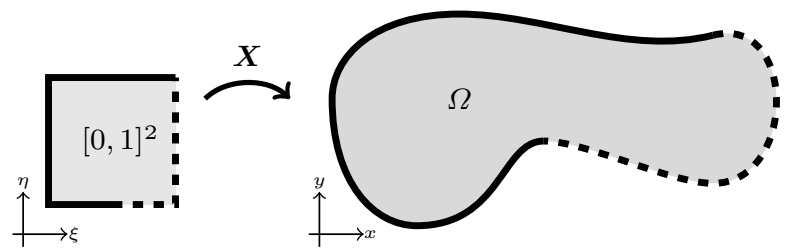

Fig. 3 Parametrization of the flow domain

For any scalar variable, including in particular the pressure $p$ and both of the velocity components $u_{1}$ and $u_{2}$, we will consider it both as a function $\bar{f}$ on physical space $\Omega$, and as a function $f$ on parameter space $[0,1]^{2}$. The gradient $\bar{\nabla} \equiv(\partial / \partial x \partial / \partial y)^{T}$ in physical space $\Omega$ is related to its counterpart $\nabla \equiv(\partial / \partial \xi \partial / \partial \eta)^{T}$ in parameter space $[0,1]^{2}$ by the following relation:

$\nabla f=\boldsymbol{J}^{T} \overline{\nabla f} \Longleftrightarrow \overline{\nabla f}=\boldsymbol{J}^{-T} \nabla f$,

where $J_{i, j}=\partial x_{i} / \partial \xi_{j}$ is the Jacobian matrix of the parametrization.

\subsection{Flow Analysis}

The governing equations (1) are solved numerically by a standard Galerkin approach based on B-splines as test and weight functions.

Approximations of the velocity $\boldsymbol{u}:[0,1]^{2} \rightarrow \mathbb{R}^{2}$ and pressure $p:[0,1]^{2} \rightarrow \mathbb{R}$ are constructed using bivariate tensor product B-splines $\mathcal{P}_{i}$ as defined above:

$\boldsymbol{u}=\sum_{j=1}^{N_{\mathrm{dof}}^{u}} \underline{\boldsymbol{u}}_{j} \mathcal{P}_{j}^{\boldsymbol{u}}+\sum_{j=N_{\mathrm{dof}}^{\boldsymbol{u}}+1}^{N_{\mathrm{var}}^{u}} \underline{\boldsymbol{u}}_{j} \mathcal{P}_{j}^{\boldsymbol{u}}$,

$p=\sum_{j=1}^{N_{\mathrm{var}}^{p}} \underline{p}_{j} \mathcal{P}_{j}^{p}$.

Here, $\underline{\boldsymbol{u}}_{j}=\left(\underline{u}_{j} \underline{v}_{j}\right)^{T} \in \mathbb{R}^{2}$ and $\underline{p}_{j} \in \mathbb{R}$ are control coefficients, and $N_{\mathrm{var}}^{\boldsymbol{u}}$ and $N_{\mathrm{var}}^{p}$ are the number of terms in the expansions for the velocity and pressure, respectively. The superscripts $\boldsymbol{u}$ and $p$ indicate that the functions refer to given knot vectors and polynomial degrees that are specific for the velocity and pressure, respectively, and the subscript $j$ denotes a single vectorized index corresponding to the two indices used in Equation (5). To ease the subsequent notation for strong enforcement of Dirichlet boundary conditions, the ordering of the terms in the velocity expansion (9a) is chosen such that the first $N_{\text {dof }}^{\boldsymbol{u}}$ functions vanish on the Dirichlet boundary $\Gamma_{D}$, whereas the last $N_{\text {fix }}^{\boldsymbol{u}}=N_{\mathrm{var}}^{\boldsymbol{u}}-N_{\mathrm{dof}}^{\boldsymbol{u}}$ functions do not.

The velocity basis functions $\mathcal{P}_{j}^{\boldsymbol{u}}$ and the pressure basis functions $\mathcal{P}_{j}^{p}$ in Equation (9) live on the parameter domain $[0,1]^{2}$. To evaluate them on the physical domain $\Omega$, we may compose them with the inverse of the geometry map, i.e., $\mathcal{P}_{j}^{\boldsymbol{u}} \circ \boldsymbol{X}^{-1}$ and $\mathcal{P}_{j}^{p} \circ \boldsymbol{X}^{-1}$, respectively. As usual in the Galerkin method, we insert the discretized fields in the governing equations (1), multiply Equation (1a) by test functions among $\mathcal{P}_{i}^{\boldsymbol{u}} \circ \boldsymbol{X}^{-1}$ for $i=1, \ldots, N_{\mathrm{dof}}^{\boldsymbol{u}}$, Equation (1b) by test functions among $\mathcal{P}_{i}^{p} \circ \boldsymbol{X}^{-1}$ for $i=1, \ldots, N_{\mathrm{dof}}^{p}$, and simplify the result using integration by parts and insertion of the Neumann boundary condition from Equation (1d). Hereby, we obtain the discretized weak or variational formulation. By interchanging the order of summation and integration, rearranging terms, and finally pulling all integrals back to parameter space, the following system of non-linear equations in the control coefficients is obtained:

$$
\begin{array}{r}
{\left[\begin{array}{ccc}
\mu \boldsymbol{K}_{1}+\rho \boldsymbol{C}_{1}(\underline{\boldsymbol{u}}) & \mathbf{0} & -\boldsymbol{G}_{1}^{T} \\
\mathbf{0} & \mu \boldsymbol{K}_{2}+\rho \boldsymbol{C}_{2}(\underline{\boldsymbol{u}}) & -\boldsymbol{G}_{2}^{T} \\
\boldsymbol{G}_{1} & \boldsymbol{G}_{2} & \mathbf{0}
\end{array}\right]\left[\begin{array}{c}
\underline{\boldsymbol{u}}_{1} \\
\underline{\boldsymbol{u}}_{2} \\
\underline{\boldsymbol{p}}
\end{array}\right]} \\
=-\left[\begin{array}{cc}
\mu \boldsymbol{K}_{1}^{\star}+\rho \boldsymbol{C}_{1}^{\star}(\underline{\boldsymbol{u}}) & \mathbf{0} \\
\mathbf{0} & \mu \boldsymbol{K}_{2}^{\star}+\rho \boldsymbol{C}_{2}^{\star}(\underline{\boldsymbol{u}}) \\
\boldsymbol{G}_{1}^{\star} & \boldsymbol{G}_{2}^{\star}
\end{array}\right]\left[\begin{array}{l}
\underline{\boldsymbol{u}}_{1}^{\star} \\
\underline{\boldsymbol{u}}_{2}^{\star}
\end{array}\right],
\end{array}
$$

or simply $\boldsymbol{M}(\boldsymbol{U}) \boldsymbol{U}=\boldsymbol{F}$, with

$$
\begin{aligned}
\underline{K}_{k, i, j} & =\iint_{[0,1]^{2}} \nabla^{T} \mathcal{P}_{i}^{u_{k}} \boldsymbol{J}^{-1} \boldsymbol{J}^{-T} \nabla \mathcal{P}_{j}^{u_{k}} \operatorname{det}(\boldsymbol{J}) \mathrm{d} \boldsymbol{\xi}, \\
\underline{C}_{k, i, j} & =\iint_{[0,1]^{2}} \mathcal{P}_{i}^{u_{k}} \boldsymbol{u}^{T}(\underline{\boldsymbol{u}}) \boldsymbol{J}^{-T} \nabla \mathcal{P}_{j}^{u_{k}} \operatorname{det}(\boldsymbol{J}) \mathrm{d} \boldsymbol{\xi}
\end{aligned}
$$




$$
\begin{aligned}
& \underline{G}_{k, i, j}=\iint_{[0,1]^{2}} \mathcal{P}_{i}^{p} \boldsymbol{e}_{k}^{T} \boldsymbol{J}^{-T} \nabla \mathcal{P}_{j}^{u_{k}} \operatorname{det}(\boldsymbol{J}) \mathrm{d} \boldsymbol{\xi}, \\
& \underline{\boldsymbol{K}}_{k}=\left[\begin{array}{ll}
\boldsymbol{K}_{k} & \boldsymbol{K}_{k}^{\star}
\end{array}\right] \quad\left(N_{\mathrm{dof}}^{u_{k}} \times\left(N_{\mathrm{dof}}^{u_{k}}+N_{\mathrm{fix}}^{u_{k}}\right)\right),
\end{aligned}
$$

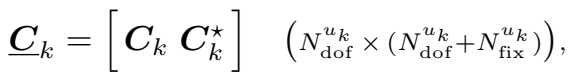

$$
\begin{aligned}
& \underline{G}_{k}=\left[\begin{array}{ll}
\boldsymbol{G}_{k} & \boldsymbol{G}_{k}^{\star}
\end{array}\right] \quad\left(N_{\mathrm{dof}}^{p} \times\left(N_{\mathrm{dof}}^{u_{k}}+N_{\mathrm{fix}}^{u_{k}}\right)\right)
\end{aligned}
$$

Here, $\boldsymbol{u}(\underline{\boldsymbol{u}})$ is given by Equation (9a), starred matrices correspond to basis functions with support on the Dirichlet boundary $\Gamma_{D}$, and starred coefficients are the corresponding coefficients that must be specified a priori to enforce the Dirichlet boundary condition in Equation (1c) strongly. Equation (10) may be solved by, e.g., an iterative Newton-Raphson method.

\subsection{Optimization}

We employ an iterative, gradient-based, non-linear optimizer to find a design that minimizes the cost function (2a) and satisfies the constraints (2b) and (2c). For sufficiently high Reynolds numbers, a gradual approach may be required in which the design successively is optimized for an increasing value of Re. Optimizing the design for a given Re, the boundary parametrization is adjusted in small steps until the design converges. In each if these design steps, the interior parametrization is firstly updated, after which the governing flow equations are solved, as described in Section 3.3 above, and the solution stored for subsequent use. To asses the quality and admissibility of the current design, the objective and constraints are then evaluated, and finally, to guide the optimization process in adjusting the current design, gradients of the cost function and the constraints with respect to the design variables are evaluated.

\subsubsection{Design Variables}

The control points $\underline{\boldsymbol{X}}_{i}$ entering the geometry parametrization in Equation (7) are the natural geometric "handles" on the flow domain, and these are therefore used as design variables for the shape optimization routine. A parametrization and its control net are sketched in Figure 4 . The design variables of the optimization are the coordinates of the control points in Figure 4a that determine the shape of the design boundary $\Gamma^{\prime}$ in Figure 4b. As also shown in Figure 4a, control points fall into three categories: The design control points are "actively" moved around in the search for the optimal shape of the design boundary, the linked control points are "passively" following the movement of the design control points to parametrize the domain interior as described below, while the fixed control points remain unaltered to keep the fixed part of the boundary unchanged.

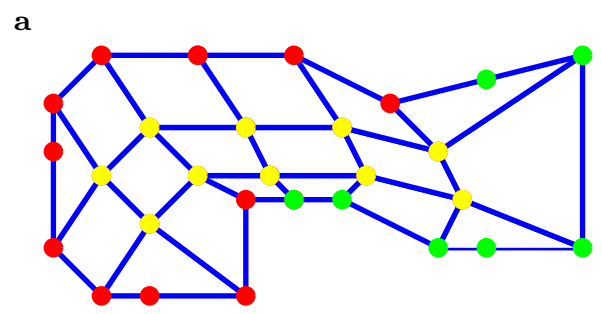

b

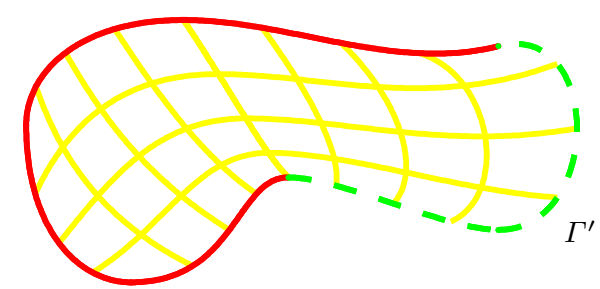

Fig. 4 a: Three types of control points: design (•), linked $(\bullet)$, and fixed $(\bullet)$. b: Image of isoparametric lines

\subsubsection{Interior Parametrization}

As the shape of flow domain is changed in the optimization process, the parametrization of its interior must be adequately updated. Referring to Figure 4, in the isogeometric framework this amounts to specifying the location of the interior control points as the location of the design control points are changed. This interior parametrization problem is a fundamental challenge in isogeometric analysis (Cohen et al 2010; Xu et al 2011). We choose to base the parametrization of the interior on the so-called Winslow functional, which is a useful measure for construction of conformal maps. Below, we briefly summarize the main points of the methodology, and refer the reader to (Gravesen et al 2010; Nguyen et al 2011) for details.

Initially, the interior control points are determined as the ones that minimize the Winslow functional, i.e. make the parametrization "as conformal as possible", while keeping the boundary constant and ensuring a valid parametrization $\operatorname{det}(\boldsymbol{J})>0$. In each design step, the interior control points are then found as those that minimizes the $2^{\text {nd }}$ order Taylor expansion of the Winslow functional based on the initial control net. The first order optimality condition for the quadratic Taylor series expansion then yields a linear problem in the interior control points. In addition, the validity of the parametrization is checked in each step by examining if $\operatorname{det}(\boldsymbol{J})>0$. If this condition is fulfilled, the optimization continues. If it is not fulfilled, the optimiza- 
tion stops, and the interior control points are found as the solution to the initial minimization problem as described above. The solution is then used as linearization point for the Taylor expansion of the Winslow functional, and the optimization is restarted.

\subsubsection{Function Evaluation}

To asses the quality and admissibility of a given design, the objective and constraint functions in Equations (3) and (2b) are evaluated in each step, based on the computed solution to the governing flow equations (10). Using the parametrization of the geometry in Equation (7) and the discretizations of the flow and pressure fields in Equation (9), we collect the control points in two $\left(N_{\mathrm{var}}^{g} \times 1\right)$ vectors $\underline{\boldsymbol{x}}$ and $\underline{\boldsymbol{y}}$, and the control coefficients in one $\left(\left(N_{\mathrm{var}}^{u}+N_{\mathrm{var}}^{v}+N_{\mathrm{var}}^{p}\right) \times 1\right)$ vector $\boldsymbol{U}=\left(\underline{\boldsymbol{u}}_{1}, \underline{\boldsymbol{u}}_{2}, \boldsymbol{p}\right)$. The mean pressure difference between two boundary segments $\gamma_{+}$and $\gamma_{-}$, the pressure variation along a boundary segment $\gamma$, the aerodynamic drag on a boundary segment $\gamma$, and the area of the domain $\Omega$, as defined in Equation (3) and (2b), may then be computed as:

$$
\begin{aligned}
C_{\Delta p} & =\mathcal{P}^{T} \underline{p}, \\
C_{\nabla p} & =\underline{\boldsymbol{p}}^{T} \mathcal{D} \underline{p} \\
C_{d} & =\mathcal{F}^{T} \boldsymbol{U}, \\
A & =\underline{\boldsymbol{x}}^{T} \mathcal{A} \underline{\boldsymbol{y}},
\end{aligned}
$$

respectively. Here, the following vectors and matrices have been defined:

$$
\begin{aligned}
\mathcal{P}= & \mathcal{P}_{\gamma_{+}}-\mathcal{P}_{\gamma_{-}} \\
\mathcal{P}_{\gamma_{i}}= & \frac{1}{L_{\gamma}} \int_{0}^{1} \mathcal{P}_{i}^{p}\|\dot{\gamma}\| \mathrm{d} \xi \\
\mathcal{D}_{i, j}= & \int_{0}^{1}\left(\boldsymbol{t}^{T} \boldsymbol{J}^{-T} \nabla \mathcal{P}_{i}^{p}\right)\left(\boldsymbol{t}^{T} \boldsymbol{J}^{-T} \nabla \mathcal{P}_{j}^{p}\right)\|\dot{\gamma}\| \mathrm{d} \xi \\
\mathcal{F}= & {\left[\begin{array}{l}
\mu \mathcal{F}_{1,1} \mu \mathcal{F}_{1,2} \\
-\mathcal{F}_{3,1}-\mathcal{F}_{3,2}
\end{array}\right] \mathcal{E}_{u} } \\
\mathcal{F}_{1,1, i}= & \int_{0}^{1}\left(2 \boldsymbol{e}_{1}^{T} \boldsymbol{n} \boldsymbol{e}_{1}^{T}+\boldsymbol{e}_{2}^{T} \boldsymbol{n} \boldsymbol{e}_{2}^{T}\right) \boldsymbol{J}^{-T} \nabla \mathcal{P}_{i}^{u}\|\dot{\gamma}\| \mathrm{d} \xi \\
\mathcal{F}_{2,1, i}= & \int_{0}^{1} \boldsymbol{e}_{2}^{T} \boldsymbol{n e}_{1}^{T} \boldsymbol{J}^{-T} \nabla \mathcal{P}_{i}^{v}\|\dot{\gamma}\| \mathrm{d} \xi
\end{aligned}
$$

$\mathcal{F}_{3,1, i}=\int_{0}^{1} \boldsymbol{e}_{1}^{T} \boldsymbol{n} \mathcal{P}_{i}^{p}\|\dot{\gamma}\| \mathrm{d} \xi$

$\mathcal{F}_{1,2, i}=\int_{0}^{1} \boldsymbol{e}_{1}^{T} \boldsymbol{n} \boldsymbol{e}_{2}^{T} \boldsymbol{J}^{-T} \nabla \mathcal{P}_{i}^{u}\|\dot{\gamma}\| \mathrm{d} \xi$,

$\mathcal{F}_{2,2, i}=\int_{0}^{1}\left(2 \boldsymbol{e}_{2}^{T} \boldsymbol{n} \boldsymbol{e}_{2}^{T}+\boldsymbol{e}_{1}^{T} \boldsymbol{n} \boldsymbol{e}_{1}^{T}\right) \boldsymbol{J}^{-T} \nabla \mathcal{P}_{i}^{v}\|\dot{\gamma}\| \mathrm{d} \xi$,

$$
\begin{aligned}
\mathcal{F}_{3,2, i} & =\int_{0}^{1} \boldsymbol{e}_{2}^{T} \boldsymbol{n} \mathcal{P}_{i}^{p}\|\dot{\gamma}\| \mathrm{d} \xi \\
\mathcal{A}_{i, j} & =\iint_{[0,1]^{2}}\left(\frac{\partial \mathcal{R}_{i}^{g}}{\partial u} \frac{\partial \mathcal{R}_{j}^{g}}{\partial v}-\frac{\partial \mathcal{R}_{i}^{g}}{\partial v} \frac{\partial \mathcal{R}_{j}^{g}}{\partial u}\right) \mathrm{d} \boldsymbol{\xi},
\end{aligned}
$$

where $\dot{\bullet} \equiv \partial \bullet / \partial \xi$ denotes the derivative with respect to the boundary parameter $\xi$, and $L_{\gamma}=\int_{0}^{1}\|\dot{\gamma}\| \mathrm{d} \xi$ is the length of the boundary. The parametric speed $\|\dot{\gamma}\| \equiv \sqrt{\dot{x}^{2}+\dot{y}^{2}}$, the unit tangent vector $\boldsymbol{t} \equiv \dot{\gamma} /\|\dot{\gamma}\|$, and the outward unit normal vector $\boldsymbol{n}$ are found by differentiating the restriction of the geometry parametrization in Equation (7) to the boundary with respect to the parameter $\xi$. These vectors and matrices are in general sparse, $\mathcal{P}, \mathcal{D}$, and $\mathcal{F}$ in particular, since only a few of the basis functions have support on the design boundary.

\subsubsection{Gradient Evaluation}

The optimization is driven by gradients of the objective and constraint functions defining the optimization problem. These sensitivities are measures of how the design variables affect the objective and constraint functions. We calculate the sensitivities analytically by a simple direct differentiation of the discretized versions of the functions in Equation (12) with respect to the coordinates of the control points that act as our design variables, and we then evaluate these gradients based on the computed solution to the governing flow equations (10). We collect the design variables in one vector $\chi$, such that $\chi=\left(\underline{x}_{1} \ldots \underline{x}_{N} \underline{y}_{1} \ldots \underline{y}_{N}\right)$, where $\left(\underline{x}_{k} \underline{y}_{k}\right)$ are the coordinates of the $k^{\text {th }}$ control point, and we let $\bullet ' \equiv \partial \bullet / \partial \chi_{k}$ denote the partial derivative with respect to the $k^{\text {th }}$ design variable. For the objectives and the constraint in Equation (12) we have:

$$
\begin{aligned}
C_{\Delta p}^{\prime} & =\mathcal{P}^{\prime T} \underline{\boldsymbol{p}}+\mathcal{P}^{T} \underline{\boldsymbol{p}}^{\prime}, \\
C_{\nabla p}^{\prime} & =\underline{\boldsymbol{p}}^{T} \mathcal{D}^{\prime} \underline{\boldsymbol{p}}+2 \underline{\boldsymbol{p}}^{T} \mathcal{\mathcal { D }} \underline{\boldsymbol{p}}^{\prime}, \\
C_{d}^{\prime} & =\mathcal{F}^{\prime T} \boldsymbol{U}+\mathcal{F}^{T} \boldsymbol{U}^{\prime}, \\
A^{\prime} & =\underline{\boldsymbol{y}}^{T} \mathcal{A} \underline{\boldsymbol{x}}^{\prime}+\underline{\boldsymbol{x}}^{T} \mathcal{A} \underline{\boldsymbol{y}}^{\prime} .
\end{aligned}
$$


The derivatives of $\underline{\boldsymbol{x}}$ and $\underline{\boldsymbol{y}}$ in Equation (14d) are trivial. The derivatives of the objective matrices/vectors $\mathcal{P}, \mathcal{D}$, and $\mathcal{F}$ in Equations (14a)-(14c) may be found by differentiation of the integrands in Equation (13). For $\mathcal{P}$ we find:

$$
\begin{aligned}
\mathcal{P}_{\gamma_{i}}^{\prime} & =\left(\frac{\left\langle\mathcal{P}_{i}^{p}\|\dot{\gamma}\|\right\rangle}{\langle\|\dot{\gamma}\|\rangle}\right)^{\prime} \\
& =\frac{\left\langle\mathcal{P}_{i}^{p}\|\dot{\gamma}\|^{\prime}\right\rangle\langle\|\dot{\gamma}\|\rangle+\left\langle\mathcal{P}_{i}^{p}\|\dot{\gamma}\|\right\rangle\left\langle\|\dot{\gamma}\|^{\prime}\right\rangle}{\langle\|\dot{\gamma}\|\rangle^{2}},
\end{aligned}
$$

where we have defined $\langle\bullet\rangle \equiv \int_{0}^{1} \bullet \mathrm{d} \xi$, and used the fact that all basis functions are independent of the design variables. Here, the derivative of the parametric speed $\|\dot{\gamma}\|$ may be found from Equation (7):

$$
\begin{aligned}
\|\dot{\gamma}\|^{\prime} & =\left(\sqrt{\dot{x}^{2}+\dot{y}^{2}}\right)^{\prime} \\
& =\frac{\dot{x} \dot{x}^{\prime}+\dot{y} \dot{y}^{\prime}}{\|\dot{\gamma}\|} \\
& =\left\{\begin{array}{l}
\frac{\dot{x}}{\|\dot{\gamma}\|} \dot{\mathcal{R}}_{k}^{g} \text { for } k=1, \ldots, N \\
\frac{\dot{y}}{\|\dot{\gamma}\|} \dot{\mathcal{R}}_{k}^{g} \text { for } k=N+1, \ldots, 2 N
\end{array}\right.
\end{aligned}
$$

Equivalent approaches may be taken for the matrices $\mathcal{D}$ and $\mathcal{F}$ in Equation (13).

The derivative of the solution $\boldsymbol{U}$ in Equations (14a)(14c) may be found by solving the linear equation system obtained by differentiation of Equation (10):

$(\boldsymbol{M}+\boldsymbol{D}) \boldsymbol{U}^{\prime}=\boldsymbol{F}^{\prime}-\boldsymbol{M}^{\prime} \boldsymbol{U}$

where

$$
\begin{aligned}
\boldsymbol{D} & =\rho\left[\begin{array}{ccc}
\boldsymbol{D}_{1,1} & \boldsymbol{D}_{1,2} & \mathbf{0} \\
\boldsymbol{D}_{2,1} & \boldsymbol{D}_{2,2} & \mathbf{0} \\
\mathbf{0} & \mathbf{0} & \mathbf{0}
\end{array}\right] \\
\boldsymbol{D}_{i, j, k, \ell} & =\iint_{[0,1]^{2}} \mathcal{P}_{k}^{u_{i}}\left(\boldsymbol{e}_{j}^{T} \boldsymbol{J}^{-T} \nabla u_{i}(\underline{\boldsymbol{u}})\right) \mathcal{P}_{\ell}^{u_{j}} \operatorname{det}(\boldsymbol{J}) \mathrm{d} \boldsymbol{\xi} .
\end{aligned}
$$

The matrix $\mathcal{J}=\boldsymbol{M}+\boldsymbol{D}$ also appears in the iterative Newton-Raphson method employed for solving the governing flow equations. For the sensitivity analysis, however, we only need to evaluate it once in each design step, based on the converged solution. The derivative of the system matrix $\boldsymbol{M}$ and vector $\boldsymbol{F}$ in Equation (17) may be found by differentiation of the integrands in Equation (11). For $\boldsymbol{K}$ we find:

$$
\begin{aligned}
K_{k, i, j}^{\prime}= & \iint_{[0,1]^{2}} \nabla^{T} \mathcal{P}_{i}^{u_{k}} \boldsymbol{J}^{-1^{\prime}} \boldsymbol{J}^{-T} \nabla \mathcal{P}_{j}^{u_{k}} \operatorname{det}(\boldsymbol{J}) \mathrm{d} \boldsymbol{\xi} \\
& +\iint_{[0,1]^{2}} \nabla^{T} \mathcal{P}_{i}^{u_{k}} \boldsymbol{J}^{-1} \boldsymbol{J}^{-T^{\prime}} \nabla \mathcal{P}_{j}^{u_{k}} \operatorname{det}(\boldsymbol{J}) \mathrm{d} \boldsymbol{\xi} . \\
& +\iint_{[0,1]^{2}} \nabla^{T} \mathcal{P}_{i}^{u_{k}} \boldsymbol{J}^{-1} \boldsymbol{J}^{-T} \nabla \mathcal{P}_{j}^{u_{k}} \operatorname{det}(\boldsymbol{J})^{\prime} \mathrm{d} \boldsymbol{\xi} .
\end{aligned}
$$

Here, the derivative of the determinant and of the inverse of the Jacobian matrix may be found by simply writing them out explicitly in terms of $x$ and $y$ and subsequently differentiating this as in Equation (16), or alternatively from the relations $\boldsymbol{J}^{-1^{\prime}}=\boldsymbol{J}^{-1} \boldsymbol{J}^{\prime} \boldsymbol{J}^{-1}$ and $\operatorname{det}(\boldsymbol{J})^{\prime}=\operatorname{det}(\boldsymbol{J}) \operatorname{tr}\left(\boldsymbol{J}^{-1} \boldsymbol{J}^{\prime}\right)$, along with differentiation of Equation (7):

$$
\boldsymbol{J}^{\prime}=\left\{\begin{array}{l}
{\left[\begin{array}{cc}
\mathcal{R}_{k ; \xi}^{g} & \mathcal{R}_{k ; \eta}^{g} \\
0 & 0
\end{array}\right] \text { for } k=1, \ldots, N} \\
{\left[\begin{array}{cc}
0 & 0 \\
\mathcal{R}_{k ; \xi}^{g} & \mathcal{R}_{k ; \eta}^{g}
\end{array}\right] \text { for } k=N+1, \ldots, 2 N}
\end{array}\right.
$$

where $\bullet_{; s} \equiv \partial \bullet / \partial s$. Equivalent approaches may be taken for the matrices $\boldsymbol{C}$ and $\boldsymbol{G}$ in Equation (11).

Finally, to account for the update of the interior parametrization, as a result of the induced movement of the linked control points when the design control points are moved, the full sensitivity is computed as $\tilde{\bullet}^{\prime}=$ $\bullet^{\prime}+\sum_{i} \chi_{i}^{l^{\prime}} \frac{\partial}{\partial \chi_{i}^{l}}$, where the summation is over linked design variables, or

$\widetilde{\nabla}_{d}=\nabla_{d}+\mathcal{W} \nabla_{l}$

where the subscripts $d$ and $l$ refer to design variables and linked variables, respectively. The matrix $\mathcal{W}_{i, j}=$ $\partial \chi_{i}^{l} / \partial \chi_{j}^{d}$ relates the linked control points to the design control points.

\subsection{Implementation Details}

The flow chart in Figure 5 sketches the most significant steps in solving the shape optimization problem in Equation (2) based on isogeometric analysis. In the initialization phase, we perform as many calculations as possible that are independent of the parametrization. In particular, all basis functions and their derivatives are evaluated in the Gauss quadrature points once and for all. Although more memory demanding, this approach greatly reduces the computational expenses, compared to evaluating the functions on the fly in each optimization step. The construction of a good initial parametrization is also vital for the optimization.

The optimization process includes an outer loop over increasing Reynolds number. As mentioned above, this is only necessary when designing shapes in higher Reynolds number flows. The entire process outlined in the flow chart may be embedded into a loop over increasing refinement of geometry and/or analysis. The optimization is performed using the SNOPT optimization package (Gill et al 2008). Standard settings for SNOPT are used, except for the step size limit which, when set relatively 


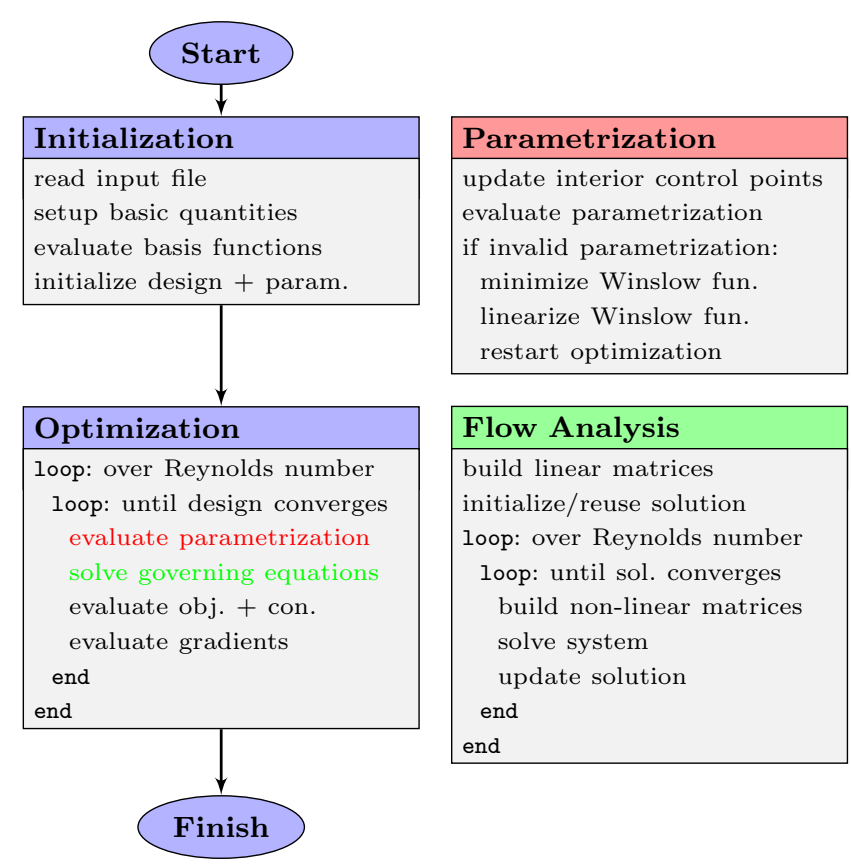

Fig. 5 Flow chart for the optimization process (left) with details of the parametrization and analysis procedures (right)

low at, e.g., $5 \%$ of the characteristic length scale of the problem, has been found to significantly improve the convergence by avoiding too large jumps in the design space. For validation purposes, the analytically computed gradients are initially checked against finite difference estimates.

On the analysis side, we use an iterative NewtonRaphson method to solve the governing non-linear Equation (10), gradually increasing the Reynolds number when this is high. In the field approximations, bi-quartic tensor product B-splines are used for the velocities and bi-cubic tensor product B-splines for the pressure, both $C^{2}$ across knots (Buffa et al 2011; Nielsen et al 2011). The finer analysis mesh (the layout of the velocity and pressure knots) is obtained by inserting knots in the coarser geometry mesh (the layout of the geometry knots). All evaluations are performed on the finer analysis mesh. Dirichlet boundary conditions are enforced strongly, while homogeneous Neumann boundary conditions are enforced weakly. All integrals are evaluated numerically using Gaussian quadrature.

\section{Regularization}

To improve the result of the shape optimization, the design space in which we look for solutions should be as large as possible. A natural way to ensure a large design space is to use many control points as design variables, although the inclusion of weights as design variables could also be considered (Qian 2010; Nagy et al 2010a,b, 2011). As the number of design control points go up, more complex shapes can be designed. This comes, however, on the cost of numerical challenges, a well-known issue from finite element methods (Bletzinger et al 2010). These challenges, and solution strategies to remedy them, are discussed in this section.

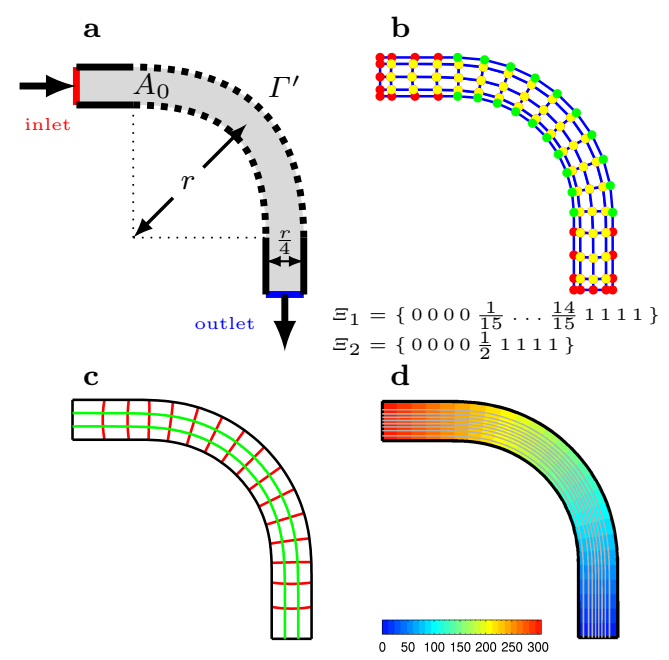

Fig. 6 Pipe bend with minimal pressure drop: design problem setup (a), initial control net (b), initial parametrization (c), and initial pressure contours and flow streamlines (d)

For the purpose of illustration, we consider a concrete example of the shape optimization problem (2)(3a), and use this as benchmark for the following tests of the regularization technique. The problem is outlined in Figure 6a. The aim is to design the shape $\Gamma^{\prime}$ of a pipe bend (dashed) to minimize the pressure drop from the inlet boundary to the outlet boundary, keeping the shape of the inlet and the outlet (solid) fixed, and with an upper bound on the area of the pipe.

We assume a parabolic horizontal velocity profile on the inlet boundary, that the velocity vanishes along the side walls, assuming no-slip conditions, and that the domain is open along the outlet boundary. We take the length scale as $r=1$, the velocity scale as $U=$ $\max \left(\left\|\boldsymbol{u}_{\text {inlet }}\right\|\right)=1$, the density as $\rho=1$, and the viscosity as $\mu=1$, assuming appropriate units are used, which yields a Reynolds number of $R e=1$ for the initial problem. The analysis is done using a total of 5,367 degrees-of-freedom.

We parametrize the pipe bend as a bi-cubic tensor product B-spline surface, and let the initial design connect the inlet and outlet by an approximate quarter annulus. The initial control net, the corresponding parametrization, and the resulting pressure distribution and streamlines of the flow through it are depicted in Figure 
$6 \mathrm{~b}-\mathrm{d}$. We use 20 control points as design variables, 10 on each of the two boundaries segments to be designed, and we allow these to move freely in both spatial dimensions, except for the four end control points, which are only allowed to move along the direction of the inlet/outlet, to keep a handle on these. As upper bound on the area, we use the initial value, i.e., $A_{\max }=A_{0}$, and we relax the lower bound, i.e., $A_{\min }=-\infty$. Since the lengths of the inlet and outlet boundaries are constant, the sensitivities in Equation (15) are greatly simplified.

\subsection{The Challenge: Clustering of Control Points}

Applying the isogeometric machinery from Section 3 to the shape optimization problem outlined above results in the optimization history depicted in Figure 7. From step $^{1} 0$ to 15 , the design control points align at first, thereby connecting the inlet and the outlet by a more or less straight segment, and decreasing the pressure drop to $\sim 74 \%$. This design reduces the length of the pipe, in intuitive accordance with the Poiseuille law. At step 44, sharper corners at the inlet and outlet are formed, but from step 44 and onwards, the shape changes only slightly, and the decrease in the pressure drop is accordingly small. The location of the control points, however, and the resulting parametrization change appreciably, though. The control points cluster and eventually fold over, resulting in an invalid parametrization after 67 steps from which the method cannot proceed.

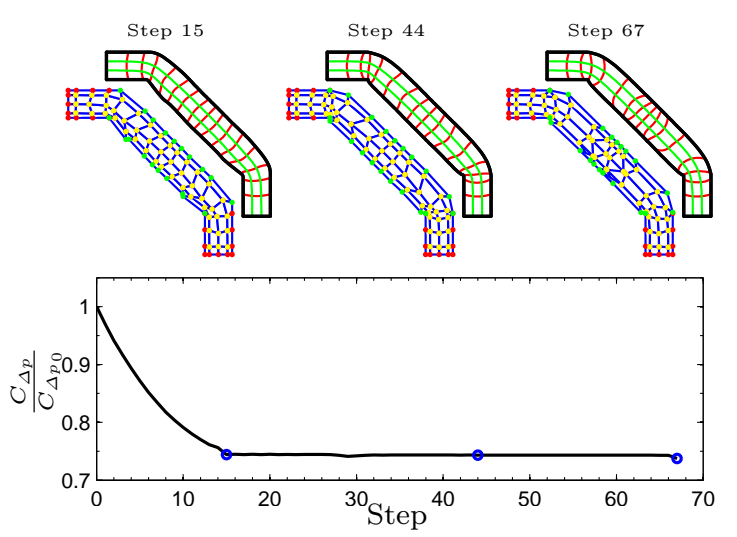

Fig. 7 Pipe bend with minimal pressure drop: objective function as a function of optimization step (bottom), and three snap shots of the control net and the associated parametrization (top)

The problem seems to arise in the second of two qualitatively different stages of the optimization: In the first stage, the optimizer finds an "optimal" shape, and

\footnotetext{
1 In SNOPT terminology, steps refer to function calls.
}

the objective function decreases significantly. In the second stage, the optimizer searches for an "optimal" parametrization of the shape, and the objective function descreases only slightly. This second stage, however, is a numerical artifact, in which the optimizer exploits the flaws in the numerical procedure, so to say, and tries to align the errors in such a way, that the numerical estimate is minimized, although the true value is not. This is the challenge in a nut-shell: when optimizing the location of many control points (for a sufficiently unconstrained problem, and with a sufficiently tight convergence criterion), they may cluster, spuriously yielding slightly lower values of the objective function on the cost of significantly worse parametrizations and less accurate analysis, which may eventually lead to a collapse of the method. The clustering of control points is a wellknown issue in isogeometric shape optimization (Wall et al 2008; Nagy et al 2011). Related numerical problems in finite element based shape optimization, and regularization techniques to address them, are also welldescribed (Bletzinger et al 2010). Below, we firstly give a brief review of some alternative ways out of the current problem, before proposing the regularization approach, in an isogeometric framework.

\subsection{Some Alternative Solution Strategies}

The first natural point of focus, when looking for remedies for the current problem, is on the optimization routine. A quick fix is simply to stop the optimization immediately after the first "shape" stage, and before the onset of the second "parametrization" stage. This could be achieved by relaxing the existing convergence criterion, or by defining some other relevant measure. However, since this approach only cures the symptoms of the problem, and not the cause of it, and due to the risk of prematurely stopping the optimization, this quick fix is, in our view, also a dirty fix.

Turning away from the optimization routine, we may focus on the problem formulation instead. An obvious solution to the problem is to reconsider the design degrees-of-freedom. As the control points align, an ambiguity is introduced, since movements of the control points along the line does not change the shape, but does change its parametrization, and thus also the numerical estimates of the integrals, and hence the objective function value, making the problem inherently ill-posed. One could then simply argue that for this particular design problem, say, four design control points on each boundary suffice. However, this is an a posteriori type of reasoning that we would like to avoid. More interesting is the idea of making this estimation of the 
necessary number of design variables dynamic, i.e., inserting and removing design control points on the fly during the optimization (Seo et al 2010b). The implementation of a flexible number of design variables in an optimization procedure, however, is far from trivial.

Preserving the number of control points, but putting constraints on their movement in the design space, poses yet another alternative. We could, for instance, constrain the design control points to move only along specified directions. In this approach, it is our duty as designers to specify "good" directions along which the control points can move, ensuring both sufficient flexibility in the design while avoiding bad parametrizations. Along the same line of thinking is the concept of putting bounds on the design variables, see e.g. (Cho and $\mathrm{Ha} 2009$ ), thus limiting the optimizer to search for a minimum in the vicinity of the initial guess only. In any case, the design space shrinks in these approaches, and the success of the optimization heavily depends on the designers choice in initial condition and constraints on the movement of the control points. A somewhat related, but much more flexible approach, is to introduce a more general constraint on the design variables. A popular choice is to put a lower bound on the distance between control points (Wall et al 2008), and although this approach does take care of the tendency of control points to cluster, it still closes the door to parts of the design space, like the sharp corners that appear at the inlet and the outlet of the pipe bend in Figure 7. Another choice is to prescribe an upper bound on a single, global measure of the shape change (Nagy et al 2011) during the optimization, thereby significantly reducing the number of constraints. Finally, very recently a regularization scheme based on a shape gradient method has been applied in an isogeometric setting (Azegami et al 2012).

\subsection{Boundary Regularization}

To avoid the problem of clustering control points and the associated fatal parametrization, and as a conceptually quite different alternative to the above methods, we suggest to use another well-known trick by regularizing the optimization problem (Mohammadi and Pironneau 2004, 2010). A similar method has also been used in an isogeometric context (Nagy et al 2010). More specifically, we suggest to add a term to the objective function that, by measuring the quality of the parametrization, prevents the undesired phenomenon. In this approach, the regularized objective function $\widetilde{C}$ is written as:

$\widetilde{C}=C+\tilde{\epsilon} R$, where $C$ is the true physical objective, here expressing the pressure drop in the pipe bend, $R$ is the artificial regularization objective, and $\tilde{\epsilon}>0$ specifies the weight of the regularization term. The aim of the optimization, when using the regularized objective, is twofold: we are not only searching for the design that minimizes the pressure drop, but also for the shape whose parametrization makes the numerical approximation of it more reliable. Thereby, we embed the construction of a good parametrization into the design optimization, and we are thus targeting the very cause of the problem.

The method poses two challenges: Firstly, it necessitates the construction of a quality measure $R$ of the parametrization, and secondly, it requires the specification of its relative importance $\tilde{\epsilon}$ in the optimization.

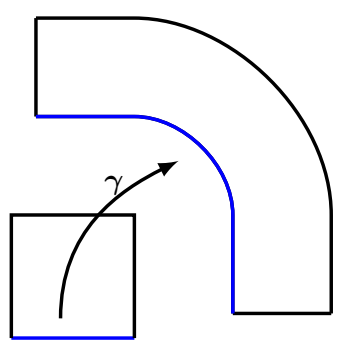

Fig. 8 Illustration of the focus of boundary regularization

For the quality measure, the focus of this study is on boundary regularization, as sketched in Figure 8. This addresses the quality of parametrization of the design boundary, which is clearly compromised in the fatal optimization history in Figure 7. We will assume that a good boundary parametrization, all other things being equal, has a constant parametric speed. Motivated by this, we define the measure as the norm squared of the parametric acceleration along the design boundaries, integrated in parameter domain:

$R=\int_{0}^{1}\|\ddot{\gamma}\|^{2} \mathrm{~d} \xi$,

where $\ddot{\bullet} \equiv \partial^{2} \bullet / \partial \xi^{2}$ denotes the second order derivative with respect to the boundary parameter $\xi$. In discretized form, it reads:

$$
\begin{aligned}
R & =\underline{\boldsymbol{x}}^{T} \boldsymbol{R} \underline{\boldsymbol{x}}+\underline{\boldsymbol{y}}^{T} \boldsymbol{R} \underline{\boldsymbol{y}}, \\
\boldsymbol{R}_{i, j} & =\int_{0}^{1} \ddot{\mathcal{R}}_{i}^{g} \ddot{\mathcal{R}}_{j}^{g} \mathrm{~d} \xi .
\end{aligned}
$$

By minimizing this measure, we bring the boundary parametrization closer to a constant-speed parametrization, and boundary regularization thus leads the optimizer towards a better boundary parametrization. The measure is computationally cheap to implement, since the matrix $\boldsymbol{R}$ only involves integrals of the second order 
derivatives of the (univariate) basis functions, and since it is quadratic in the design variables, the sensitivities may be straightforwardly computed.

An important challenge in the methodology is the specification of a suitable weight $\tilde{\epsilon}$ of the regularization. This challenge is similar in nature to the one associated with specifying a suitable minimal distance between control points (Wall et al 2008), or a maximal shape change norm (Nagy et al 2011). The specification may be partly facilitated by estimating the initial ratio between the physical objective $C_{0}$ and the regularization objective $R_{0}$ :

$\tilde{\epsilon}=\frac{\left|C_{0}\right|}{\left|R_{0}\right|} \epsilon$,

assuming $R_{0} \neq 0$, and that this ratio does not change too much with the design. Taking $\epsilon=1$ yields identical initial numerical values for the physical and the regularization terms in Equation (22). Usually, a value $\epsilon \ll 1$ is therefore anticipated. The smaller the $\epsilon$, the closer we get to the original optimization problem, but, on the other hand, the more we weaken the regularization and its stabilizing influence on the parametrization and the convergence.

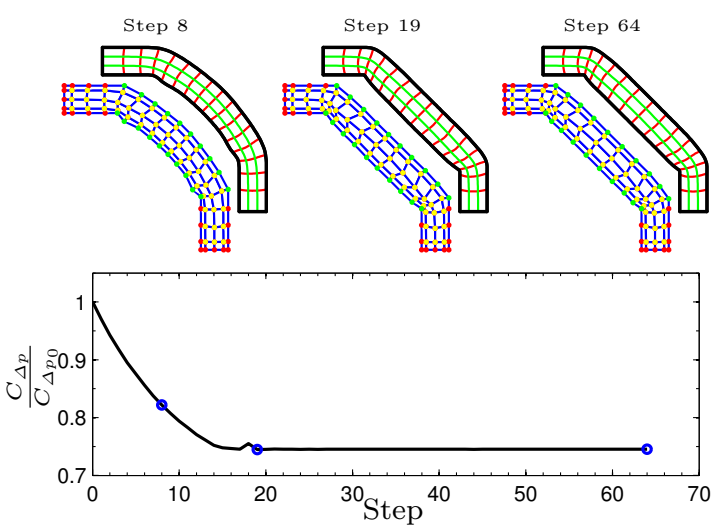

Fig. 9 Pipe bend with minimal pressure drop: regularized optimization history (bottom), and snap shots of the control net and the associated parametrization (top)

We apply the regularized isogeometric shape optimization method to the current design problem, thus minimizing the regularized pressure drop (22)-(23) throu the pipe bend using the weight $\epsilon=10^{-2}$. The optimal design is reached after 64 steps, at which point the pressure drop is decreased to $74.5 \%$. The optimization history is shown in Figure 9. Here, it is worth noticing that the optimal design is quite close to the design from which the original formulation drifts off, cf. Figure 7 , that the difference in the minimal pressure drop between the designs is small, and, most importantly, that the parametrization is much better in this regularized formulation, thereby making the analysis more reliable. The effect of the regularization is clearly seen from the intermediate design in step 19, to the converged design in step 64 . The control points spread out along the line, and the concentration of control points is shifted away from the straight central part, towards the curved parts at the inlet and the outlet. This is also where the geometry, and hence the flow analysis, is most challenging, due to the presence of sharp corners that form as a result of coalescing control points. The resulting pressure field and streamlines are shown in Figure 10. The optimized design is very similar to the topology optimized design with minimal energy dissipation (Gersborg-Hansen et al 2005).

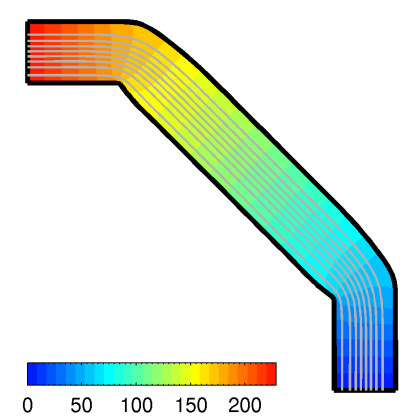

Fig. 10 Pipe bend with minimal pressure drop: optimized pressure contours and flow streamlines

To examine the effect of the regularization in greater detail, we solve the problem for a range of regularization weights $\epsilon \in\left[10^{-3}, 10^{-1}\right]$. Figure 11 shows how the optimized pressure drop, the required number of steps, and the optimal design vary with the regularization weight. When the regularization is strong, the optimization converges quickly to a smoother design with a higher pressure drop. As the regularization is decreased, more steps are required to reach designs with locally higher curvature and smaller pressure drops. A stagnation point in the pressure drop curve is observed, associated with the formation of the sharp corners at the inlet and the outlet, such that the optimized pressure drop only falls off slightly for $\epsilon \leq 3 \cdot 10^{-2}$. In addigh tion, the number of steps is likewise relatively constant for $3 \cdot 10^{-3} \leq \epsilon \leq 3 \cdot 10^{-2}$. A regularization weight in this range thus seems appropriate in this example. The results, however, are not critically sensitive to the value used, cf. Figure 11.

We conclude this section by mentioning that a range of other regularization measures could be considered. Among these, we have found that similar effects as those presented above may be obtained by minimizing 


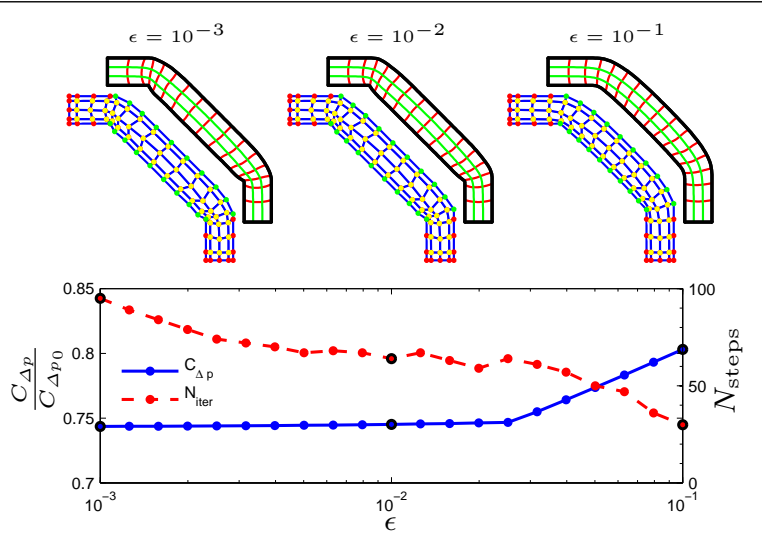

Fig. 11 Pipe bend with minimal pressure drop: optimized pressure drop and required number of steps as a function of regularization weight $\epsilon$ (bottom), and optimal design for three values of $\epsilon$ (top)

the scalar product of the tangent and the acceleration, the variance of the Jacobian determinant, or the variance of the parametric speed, all evaluated along the design boundary. Furthermore, the regularization measures could alternatively be implemented as constraints, but such investigations have been outside the scope of this study.

\section{Applications}

In the following, we apply the isogeometric shape optimization methodology for fluid mechanical design problems to two additional numerical examples: Section 5.1 studies a design problem based on Taylor-Couette flow and investigates how the optimization is affected by the number of geometric design variables, while Section 5.2 studies the minimal drag body design problem, and investigates how the optimization responds to variations in the analysis resolution, initial design and Reynolds number.

\subsection{Body with Uniform Pressure Distribution}

We consider the shape optimization problem (2)-(3b) outlined in Figure 12a. The aim is to design the boundary $\Gamma^{\prime}$ of a body of given area $A_{0}$, placed in a circular fluid container of radius $r$ whose outer boundary rotates at a constant rate, to make the pressure distribution along $\Gamma^{\prime}$ as uniform as possible, i.e., to minimize the pressure variation $C_{\nabla p}$ along $\Gamma^{\prime}$. From symmetry considerations, the pressure is constant along the boundary when a disk is placed in the center. Furthermore, analytical solutions to the governing NavierStokes equations for this so-called Taylor-Couette flow problem is well-known from the literature. A circle enclosing the specified area and with its center in the center of the container is therefore a solution to the shape optimization problem. In the following, we investigate how well the isogeometric shape optimization methodology is able to reproduce this.
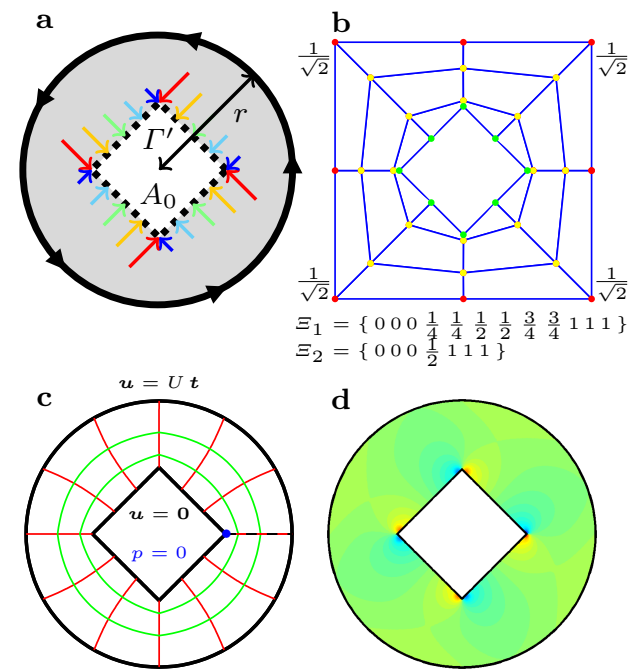

Fig. 12 Body with uniform pressure distribution: design problem setup (a), initial control net, knot vectors, and nonunitary weights (b), initial parametrization (c), and initial pressure field (d)

To represent the outer perimeter as an exact circle, which is of paramount importance when specifying boundary conditions, we parametrize the geometry using quadratic NURBS, while ordinary B-splines are used elsewhere. As initial design, we use a square placed in the middle, which is an intentionally bad initial design. The control net, knot vectors and weights are shown in Figure 12b, and the corresponding parametrization is visualized in Figure 12c. The patch is attached to itself along the dashed line, resulting in an additional $C^{0}$-continuity here. The velocity field is specified as purely tangential along the outer, moving perimeter, and as vanishing on the inner, steady boundary, assuming no-slip conditions. Since full Dirichlet boundary conditions are prescribed for the velocity field, we set the pressure to zero in an arbitrary point. We take the initial area as $A_{0}=2$, the radius of the outer perimeter as $r=2$, the velocity scale as $U=1$, the density as $\rho=1$, and the viscosity as $\mu=1$, assuming again appropriate units, which again yields a Reynolds number of $\operatorname{Re}=1$. The initial pressure field is depicted in Figure 12 d. In this, the $C^{0}$-continuities are invisible to the naked eye. We take the constraint on the area as the initial value, i.e., $A_{\max }=A_{\min }=\pi r^{2}-A_{0}$, and a one-step approach is employed for the Reynolds num- 
ber. To resolve the rotational symmetry, the left-most control point is allowed only to move horizontally. It turns out that this problem is sufficiently constrained to prevent control points from clustering, and we may solve it without regularization.
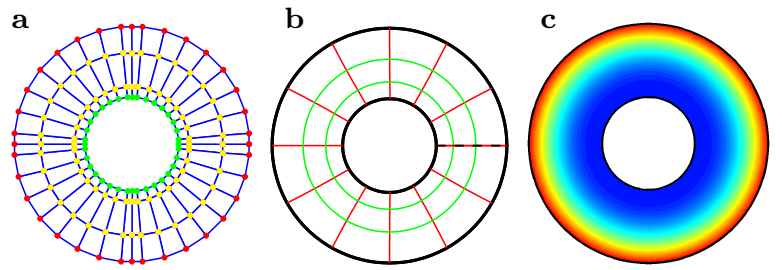

Fig. 13 Body with uniform pressure distribution: optimized control net (a), parametrization (b), and pressure field (c) for 36 design control points

We consider three consecutive refinements of the coarse geometry described above, obtained by uniform knot refinement along the tangential direction, thereby representing the design boundary $\Gamma^{\prime}$ by $8,12,20$, and 36 control points, respectively. We keep the analysis mesh fixed, using a total of 5,715 degrees-of-freedom. We solve the design problem for each of these four geometric models, using in turn the optimized coarser design as initial design for the finer optimization. The results for the finest geometry approximation are shown in Figure 13. The optimal design is reached in a total of 1032 steps, and the pressure variation is decreased by a factor of $\sim 10^{13}$. The optimal control net is shown in (a), the corresponding parametrization in (b), and the corresponding pressure distribution in (c). The pressure field along the optimized boundary is significantly more uniform than the initial one shown in Figure 12d, and the optimized boundary is seen to approximate a circle very accurately.

To examine more closely the effect of enlarging the design space by the use of more design control points, Figure 14a shows the pressure distribution along the optimized boundary when using $8,16,20$, and 36 design control points. Also shown, in Figure 14b, is a comparison of the lower part of the optimized design boundaries to the exact circle. As is evident from the figure, the more control points we use, the more uniform the pressure distribution we obtain, and the better the approximation to the exact circle we find. This is quantitatively supported by the numerical values listed in Table 1, showing that, as more design control points ( $1^{\text {st }}$ column) are used, both the pressure variation $\left(2^{\text {nd }}\right.$ column), and the discrepancy of the design boundary from the circle of radius $r_{0}=\sqrt{A_{0} / \pi}$ ( $3^{\text {rd }}$ column $)$ tend towards zero. Here, we have estimated the discrepancy of the design boundary from the circle of radius $r_{0}$ by

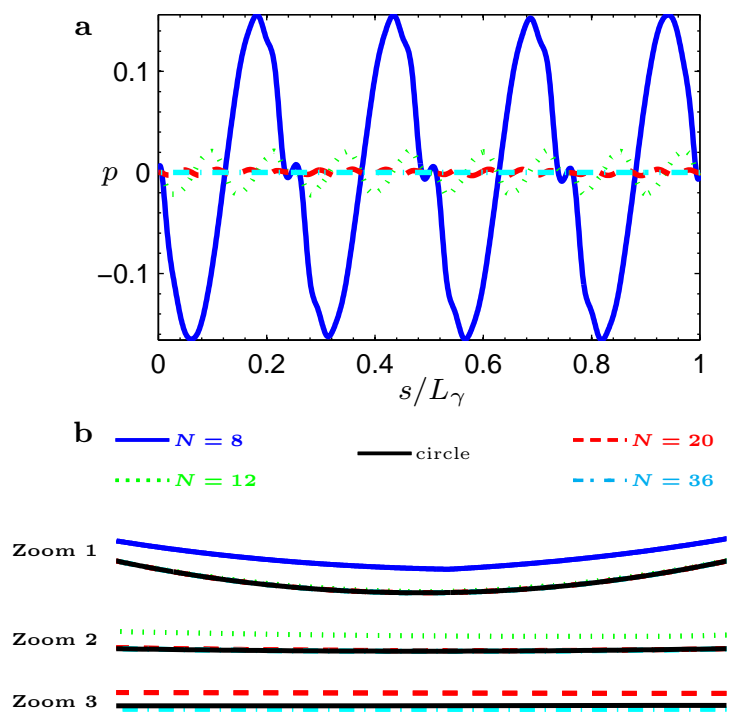

Fig. 14 Body with uniform pressure distribution: comparison of pressure distributions along the optimized boundaries (a), and comparison of the optimized boundaries to the exact circle in three zooms (b)

Table 1 Body with uniform pressure distribution: comparison of objective function, discrepancy from the exact circle, and number of steps for different numbers of design control points

\begin{tabular}{cccc}
\hline$N_{\text {design }}$ & $C_{\nabla p} / C_{\nabla p 0}$ & $\epsilon_{\text {circle }}$ & $N_{\text {steps }}$ \\
\hline 8 & $8.1 \cdot 10^{-4}$ & $6.3 \cdot 10^{-2}$ & 131 \\
12 & $5.1 \cdot 10^{-5}$ & $3.7 \cdot 10^{-3}$ & 127 \\
20 & $3.3 \cdot 10^{-6}$ & $2.5 \cdot 10^{-4}$ & 344 \\
36 & $1.4 \cdot 10^{-17}$ & $2.1 \cdot 10^{-5}$ & 430 \\
\hline
\end{tabular}

the measure:

$\epsilon_{\text {circle }}^{2}=\int_{\gamma}\left(\frac{x^{2}+y^{2}}{r_{0}^{2}}-1\right)^{2} \mathrm{~d} s$.

With 36 design control points, this error is $2.1 \cdot 10^{-5}$.

\subsection{Body with Minimal Drag}

We consider the shape optimization problem (2)-(3c) outlined in Figure 15a. The boundary $\Gamma^{\prime}$ of a body with given minimal area $A_{0}$ traveling at constant speed $U$ through a fluid is designed to minimize the $\operatorname{drag} C_{d}$ it experiences as the fluid flows past it.

Symmetry is assumed around the line along which the body travels, and only the upper half of the problem is considered. To facilitate the implementation of boundary conditions, and to achieve some local refinement close to the body, this half space is modeled using two patches, as shown in Figure 15b (top). The computational domain extends $20 r$ upstream, $20 r$ sidewards, and $40 r$ downstream, as depicted in Figure 15b 


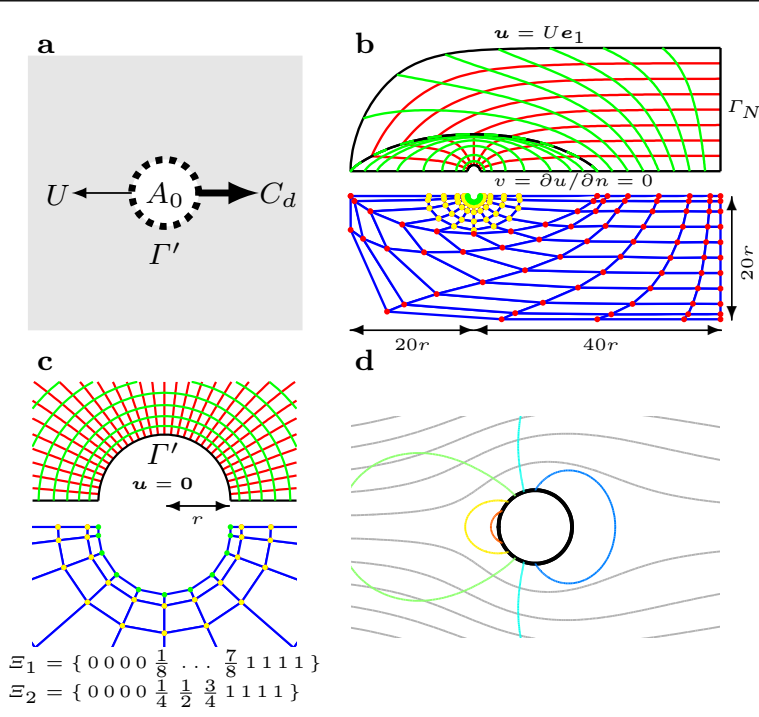

Fig. 15 Body with minimal drag: design problem setup (a), initial control net, initial parametrization, domain dimensions, boundary conditions, and knot vectors ( $\mathbf{b}$ and $\mathbf{c}$ ), and initial pressure contours and flow streamlines for $\operatorname{Re}=1(\mathbf{d})$

(bottom). The governing equations are solved in the comoving inertial system in which the body is at rest. For the boundary conditions, we assume no-slip along the design boundary $\Gamma^{\prime}$, that the flow is undisturbed along the upstream truncation boundary, that the downstream truncation boundary is open, and that the fields are symmetric around the lower truncation boundary, as sketched in Figures 15b-c (top). The flow speed, the density and the viscosity are set to $U=1, \rho=1$ and $\mu=1$, respectively, assuming again appropriate units, and the design boundary $\Gamma^{\prime}$ is initialized as an approximate half circle of radius $r=1$, as depicted in Figure 15c (top). This corresponds to a Reynolds number of $\mathrm{Re}=1$. Cubic B-splines are employed for the geometry parametrization, and the analysis uses a total 25,452 degrees-of-freedom. The initial control net is shown in Figures 15b-c (bottom). We use 11 design control points, and take $A_{0}=\pi$ as lower bound on the area of the body, i.e., $A_{\max }=A_{\Omega}-A_{0} / 2$, where $A_{\Omega}$ denotes the area of the computational domain $\Omega$, and $A_{\min }=-\infty$. The end control points are allowed only to move horizontally and symmetrically, to ensure that the domain is appropriately defined, and to resolve the translational symmetry of the problem. To prevent the control net from folding over at the leading and trailing edges in particular, boundary regularization with weight $\epsilon=10^{-2}$ is employed.

Referring to the configuration described above, we firstly investigate how variations in initial design and analysis resolution affect the optimization convergence and results. From the baseline configuration (a), we consider two experiments with the initial design per- turbed $(b-c)$, and two experiments with the analysis resolution perturbed $(\mathrm{d}-\mathrm{e})$, keeping all other parameters fixed in each experiment. The initial designs for these five different configurations are shown in Figure $16 \mathrm{a}$, and their characteristics are summarized in columns $1-3$ of Table 2 . The optimization results are

Table 2 Body with minimal drag for different initial designs (Start) and analysis resolutions $\left(N_{\mathrm{var}}\right)$ : Number of steps $\left(N_{\text {step }}\right)$, length $(L)$, and height $(H)$ of the optimized designs

\begin{tabular}{cccccc}
\hline Exp. & Start & $N_{\text {var }}$ & $N_{\text {step }}$ & $L / 2$ & $H / 2$ \\
\hline a & circle & 25,452 & 65 & 1.81 & 0.62 \\
b & square & 25,452 & 46 & 1.75 & 0.62 \\
c & ellipse & 25,452 & 80 & 1.80 & 0.62 \\
d & circle & 11,802 & 83 & 1.80 & 0.62 \\
e & circle & 44,286 & 99 & 1.82 & 0.62 \\
\hline
\end{tabular}

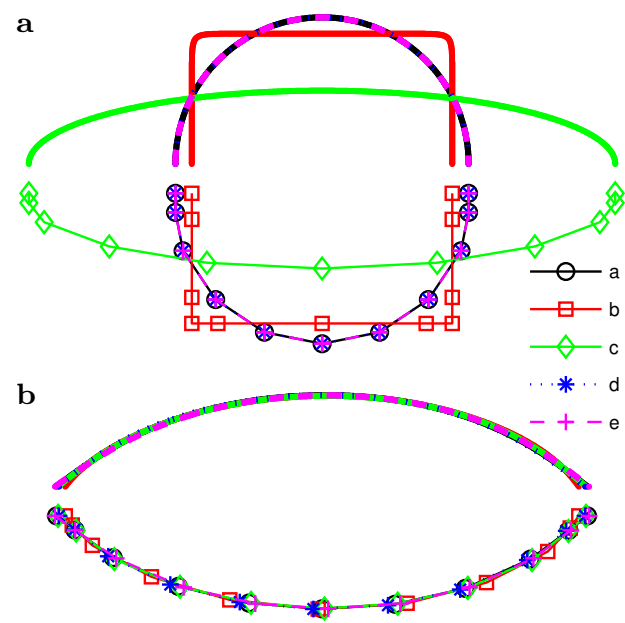

Fig. 16 Body with minimal drag for different initial designs and analysis resolutions. a: Initial design boundary (top) and control points (bottom). b: Optimized design boundary (top) and control points (bottom)

shown in Figure 16b, and the main features are summarized in columns $4-6$ of Table 2 . From these, we notice that the optimized designs are quite similar, both when the initial design is perturbed, and when the analysis resolution is perturbed. Considering the configurations all together, the largest relative difference in the optimal length of the body is $\sim 4 \%$ from configuration $\mathrm{b}$ to configuration e. The number of design steps, however, varies considerably. Here, the finer the analysis resolution, the more design steps are used. For variations in the initial design, the picture is more blurred. The remarkably few steps used in configuration b is likely due to the absence of updates of the interior parametri- 
zation during the optimization, which is done once in the other configurations. Considering the configurations all together, the number of design steps varies by more than a factor of 2 from configuration $b$ to configuration e.

Referring again to the baseline configuration described above, we now investigate how the optimal design depends on the Reynolds number, by adjusting the viscosity $\mu$. We solve the shape optimization problem for four consecutive Reynolds numbers $\operatorname{Re} \in\{1,10,40,100\}$ based on the initial baseline design, using again the optimized design of the lower-Re problem as initial design for the higher-Re problem. After a total of $65+$ $69+60+65=259$ design steps, the optimization converges. To illustrate how the design varies with the

a

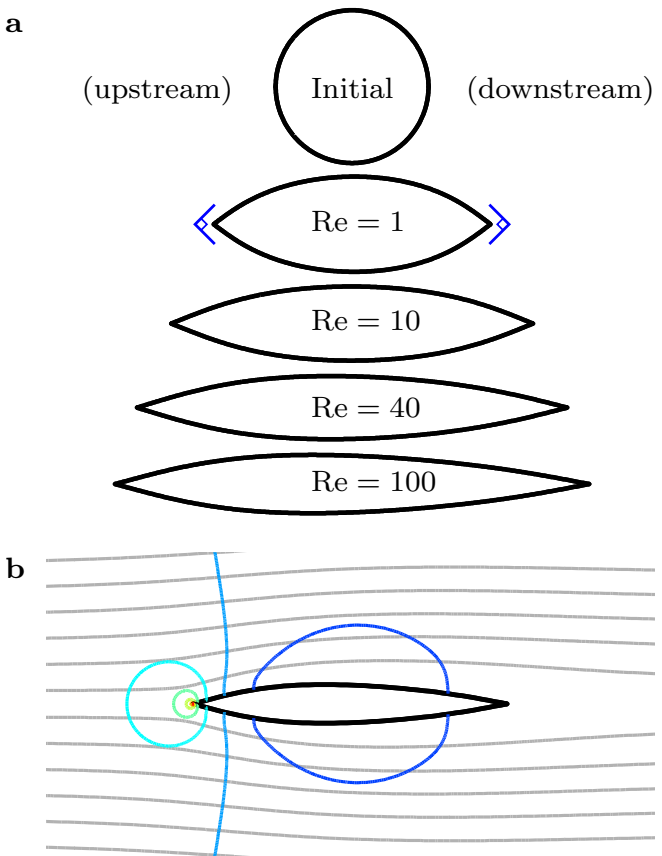

Fig. 17 Body with minimal drag for different Reynolds numbers: initial and optimized shapes (a), and optimized pressure contours and flow streamlines for $\operatorname{Re}=100(\mathbf{b})$

Table 3 Body with minimal drag for different Reynolds numbers: length $(L)$, height $(H)$, widest location $\left(x_{w}\right)$, and relative decrease in $\operatorname{drag} r=\left(C_{d}^{\text {initial }}-C_{d}^{\text {optimal }}\right) / C_{d}^{\text {initial }}$ for the initial and optimized shapes

\begin{tabular}{lcccc}
\hline $\operatorname{Re}$ & $L / 2$ & $H / 2$ & $x_{w}$ & $r$ \\
\hline Initial & 1.00 & 1.00 & 0.00 & - \\
1 & 1.81 & 0.62 & 0.03 & $7.7 \%$ \\
10 & 2.37 & 0.49 & -0.01 & $2.3 \%$ \\
40 & 2.81 & 0.41 & -0.29 & $1.2 \%$ \\
100 & 3.10 & 0.38 & -0.52 & $0.4 \%$ \\
\hline
\end{tabular}

Reynolds number, the initial and the four optimized shapes are compared in Figure 17a, and the characteristics of the shapes are summarized in Table 3. A considerable change in the design is seen as the Reynolds number is increased: for low values, a body shaped like a rugby ball is optimal, while for higher values, a more slender design is optimal, with a slightly thicker upstream part than downstream part. The latter relates well to the increase in the significance of the form drag, , and the decrease in the significance of the skin friction drag, as Re increases. The pressure and flow fields around the optimized shape for $\mathrm{Re}=100$ are depicted in Figure 17b.

In the present context, minimizing the drag on the body is equivalent to minimizing the energy dissipation in the flow past it (Mohammadi and Pironneau 2010), and we may compare the results for these two types of problems. Firstly, for Reynolds number $\mathrm{Re}=1$, the angles of the leading edge (upstream) and the trailing edge (downstream) compare well to the theoretically predicted value of $90^{\circ}$, while for higher Reynolds numbers, the present shapes are clearly more cusped (Pironneau 1973, 1974). For Reynolds number $\mathrm{Re}=1$, the present shape compare well qualitatively to the numerical results obtained in (Katamine et al 2005), while for Reynolds numbers Re $>1$, they differ significantly from their ovoid with the upstream part slimmer than the downstream part. Consistently better qualitative correspondence is found with the numerical results in (Kim and Kim 1995), although the present shapes are slightly longer, thinner, and more ovoid than their elliptic shapes.

\section{Conclusions}

In this work, we have applied isogeometric analysis to shape optimization problems in fluid mechanics. The numerical method uses NURBS and B-splines from computer aided design both as analysis tool in a finiteelement-like manner to solve the governing steady-state, incompressible Navier-Stokes equations in two spatial dimensions, and as design tool to find optimal shapes by adjusting the control points using a gradient-based numerical optimization package.

Using the integral of the norm squared parametric acceleration along the design boundary as a measure of the quality of the boundary parametrization, we have established a cheap, flexible and efficient regularization technique to avoid inappropriate parametrizations during optimization, by simply adding it to the objective function. The regularization embeds the construction of a good parametrization into the design optimization, allowing the designer to search for shapes in a large 
design space, with little a priori knowledge on the optimal design. Its greatest challenge lies in the choice of the regularization weight.

To emphasize the robustness of the proposed methodology, we have used it in three different numerical examples: first a pipe bend was designed to minimize the pressure drop of the flow through it, then a body at rest in a circular fluid container with rotating boundary was designed to obtain a uniform pressure distribution along its boundary, and finally a body traveling at constant speed through a fluid was designed to minimize the drag from the flow past it. Through the first example we discussed some advantages and challenges for the boundary regularization technique. Through the second example we showed that progressively better approximations of a known solution was achieved when more design control points were used. Through the third example we demonstrated that practically identical shapes were obtained for different initial designs and analysis resolutions, whereas significantly different shapes were obtained when varying the Reynolds number.

This work serves as a proof-of-concept of isogeometric shape optimization as a viable numerical method for accurate design of complex shapes in engineering problems within fluid mechanics. Future studies within the field could address flows with higher Reynolds numbers, extensions to three spatial dimensions and nonstationary conditions, fluid-structure interactions, local mesh refinements, regularization measures, and methods for parametrization of the domain interior during design of the domain boundary, to name but a few relevant subjects.

\section{Acknowledgment}

The authors would like to thank Allan Roulund Gersborg, Burmeister \& Wain Energy A/S, Denmark, Thomas A. Grandine and Thomas A. Hogan, The Boeing Company, USA, and Mathias Stolpe, DTU Wind Energy, Denmark, for conceptual support and fruitful discussions during the course of this work. We also thank two anonymous reviewers for valuable comments on the manuscript.

\section{References}

Akkerman I, Bazilevs Y, Calo VM, Hughes TJR, Hulshoff S (2010) The role of continuity in residual-based variational multiscale modeling of turbulence. Comput Mech 41:371378

Azegami H, Fukumoto S, Aoyama T (2012) Shape optimization of contiua using NURBS as basis funtions. Struct Multidisc Optim DOI 10.1007/s00158-012-0822-4
Bazilevs Y, Hughes TJR (2008) NURBS-based isogeometric analysis for the computation of flows about rotating components. Comput Mech 43:143-150

Bletzinger KU, Firl M, Linhard J, Wüchner R (2010) Optimal shapes of mechanically motivated surfaces. Comput Methods Appl Mech Engrg 199:324-333

Buffa A, de Falco C, Sangalli G (2011) IsoGeometric Analysis: Stable elements for the 2D Stokes equation. Int J Numer Meth Fluids 65:1407-1422

Cho S, Ha SH (2009) Isogeometric shape design optimization: exact geometry and enhanced sensitivity. Struct Multidisc Optim 38:53-70

Cohen E, Martin T, Kirby RM, Lyche T, Riesenfeld RF (2010) Analysis-aware modeling: understanding quality considerations in modeling for isogeometric analysis. Comput Methods Appl Mech Engrg 199:334-356

Cottrell JA, Hughes TJR, Bazilevs Y (2009) Isogeometric Analysis: Toward Integration of CAD and FEA. John Wiley and Sons

Donea J, Huerta A (2003) Finite element methods for flow problems. John Wiley and Sons

Gersborg-Hansen A, Sigmund O, Haber RB (2005) Topology optimization of channel flow problems. Struct Multidisc Optim 30(3):181-192

Gill PE, Murray W, Saunders A (2008) User's guide for SNOPT version 7: Software for large-scale nonlinear programming. http://www.cam.ucsd.edu/ peg/papers/sndoc7.pdf. Accessed 25 January, 2012.

Gravesen J, Evgrafov A, Gersborg AR, Nguyen DM, Nielsen PN (2010) Isogeometric analysis and shape optimisation. In: Eriksson A, Tibert G (eds) Proceedings of NSCM-23: the 23rd Nordic Seminar on Computational Mechanics, pp 14-17

Ha SH, Choi KK, Cho S (2010) Numerical method for shape optimization using T-spline based isogeometric method. Struct Multidisc Optim 42:417-428

Hassani B, Khanzadi M, Tavakkoli SM (2012) An isogeometrical approach to structural topology optimization by optimality criteria. Struct Multidisc Optim 45:223-233

Hughes TJR, Cottrell JA, Bazilevs Y (2005) Isogeometric analysis: CAD, finite elements, NURBS, exact geometry and mesh refinement. Comput Methods Appl Mech Engrg 194:4135-4195

Ivorra B, Hertzog DE, Mohammadi B, Santiago JS (2006) Semi-deterministic and genetic algorithms for global optimization of microfluidic protein-folding devices. Int J Numer Meth Eng 66:319-333

Katamine E, Azegami H, Tsubata T, Itoh S (2005) Solution to shape optimization problems of viscous flow fields. Int J Comput Fluid D 19(1):45-51

Kim DW, Kim MU (1995) Minimum drag shape in twodimensional viscous flow. Int J Numer Meth Fl 21:93-111

Li K, Qian X (2011) Isogeometric analysis and shape optimization via boundary integral. Comput Aided Design 43:1427-1437

Mohammadi B, Pironneau O (2004) Shape optimization in fluid mechanics. Annu Rev Fluid Mech 36:255-279

Mohammadi B, Pironneau O (2010) Applied Shape Optimization for Fluids, 2nd edn. Oxford Science Publications

Nagy AP, Abdalla MM, Gürdal Z (2010) Design of anisotropic composite shells using an isogeometric approach. In proceedings of the 13th AIAA/ISSMO Multidisciplinary Analysis Optimization Conference, Fort Worth TX, USA

Nagy AP, Abdalla MM, Gürdal Z (2010a) Isogeometric sizing and shape optimization of beam structures. Comput 
Methods Appl Mech Engrg 199:1216-1230

Nagy AP, Abdalla MM, Gürdal Z (2010b) On the variational formulation of stress constraints in isogeometric design. Comput Methods Appl Mech Engrg 199:2687-2696

Nagy AP, Abdalla MM, Gürdal Z (2011) Isogeometric design of elastic arches for maximum fundamental frequency. Struct Multidisc Optim 43:135-149

Nguyen DM, Evgrafov A, Gersborg AR, Gravesen J (2011) Isogeometric shape optimization of vibrating membranes. Comput Methods Appl Mech Engrg 200:1343-1353

Nielsen PN, Gersborg AR, Gravesen J, Pedersen NL (2011) Discretizations in isogeometric analysis of Navier-Stokes flow. Comput Methods Appl Mech Engrg 200:3242-3253

Painchaud-Oullet S, Tribes C, Trepanier JY, Pelletier D (2006) Airfoil shape optimization using a nonuniform rational B-spline parametrization under thickness constraint. IAAA Journal 44(10):2170-2178

Piegl L, Tiller W (1995) The NURBS Book. Springer

Pironneau O (1973) On optimum profiles in Stokes flow. J Fluid Mech 59:117-128

Pironneau O (1974) On optimum design in fluid mechanics. J Fluid Mech 64:97-110

Qian X (2010) Full analytical sensitivities in NURBS based isogeometric shape optimization. Comput Methods Appl Mech Engrg 199:2059-2071

Qian X, Sigmund O (2011) Isogeometric shape optimization of photonic crystals via Coons patches. Comput Methods Appl Mech Engrg 200:2237-2255

Seo YD, Kim HJ, Youn SK (2010a) Isogeometric topology optimization using trimmed spline surfaces. Comput Methods Appl Mech Engrg 199:3270-3296

Seo YD, Kim HJ, Youn SK (2010b) Shape optimization and its extension to topological design based on isogeometric analysis. Int J Solids Struct 47:1618-1640

Wall WA, Frenzel MA, Cyron C (2008) Isogeometric structural shape optimization. Comput Methods Appl Mech Engrg 197:2976-2988

Xu G, Mourrain B, Duvigneau R, Galligo A (2011) Parameterization of computational domain in isogeometric analysis: Methods and comparison. Comput Methods Appl Mech Engrg 200(2324):2021-2031 